\title{
A Game-Theoretic Approach for Exploiting Multiuser Diversity in Cooperative Slotted Aloha
}

\author{
Dandan Wang, Student Member, IEEE, Cristina Comaniciu, Member, IEEE, Hlaing Minn, Senior Member, IEEE, \\ and Naofal Al-Dhahir, Fellow, IEEE
}

\begin{abstract}
Multiuser diversity gains can be achieved by assigning channels to users with better channel quality in multiuser systems. To avoid the extensive information exchange required for centralized approaches, we propose a distributed fair pricing strategy for a slotted Aloha system in which users act selfishly to improve their own utilities for both the collision model and the multipacket reception (MPR) model. Based on a gametheoretic framework, we show that multiuser diversity gains can be achieved by appropriately designing the Nash equilibrium thresholds for the selfish users to preserve the throughput and revenue achieved in the classical slotted Aloha systems. The network enforces fairness among different users by employing a pricing policy that favors equal access probabilities. Our simulation results show that significant multiuser diversity gains are achieved in terms of energy consumption and/or spectral efficiency.
\end{abstract}

Index Terms-

\section{INTRODUCTION}

A Fundamental characteristic of wireless mobile communication systems is random channel fading. Traditionally, diversities in the time, space, and frequency domains are exploited to combat the detrimental effects of channel fading. However, instead of mitigating fading on each user's channel, multiuser diversity has been proposed to maximize the total information-theoretic capacity in the context of multiuser communications [1] [2]. The basic idea of multiuser diversity is to exploit the randomness of fading channels among different users. The larger the dynamic range of channel fluctuations and the number of users, the larger the available multiuser diversity gain is. In a centralized downlink system, the access point assigns the channel to the user with the best instantaneous channel gain as in [1] and [3]. However, centralized scheduling schemes require significant information exchange between users and the access point. Consequently, a distributed access scheme which reduces this information exchange but can still utilize the multiuser diversity is very desirable. In [4], a distributed channel-aware slotted Aloha

Manuscript received May, 3, 2007; revised October 22, 2007 and February, 29, 2008; accepted April, 14, 2008. The associate editor coordinating the review of this letter and approving it for publication was Prof. Il-Min Kim.

The authors are with the Dept. of Electrical Engineering, University of Texas at Dallas, Richardson, TX, 75083, USA. emails: \{dxw053000, hlaing.minn, aldhahir\}@utdallas.edu and the Dept. of Electrical Engineering, Stevens Institute of Technology, Hoboken, NJ, 07094, USA. email: ccomanic@stevens.edu

The work of this author was supported in part by NSF grant number CNS0435297. The work of these authors was supported in parts by the Erik Jonsson School Research Excellence Initiative, the University of Texas at Dallas, USA, and the National Science Foundation (NSF) under Contracts CCF 04-30654 and DMS 05-28010. The material in this paper has been presented in part in IEEE ICC, Glasgow, Scotland, June, 2007.

Digital Object Identifier 10.1109/TWC.2008.070464. scheme is proposed in which each user utilizes only his own channel information and assumes that users behave in a predictable manner which is set by the network. However, we note that in a distributed system, users may act selfishly to maximize their own performance.

In this paper, we use a game-theoretic approach to design the pricing-based medium access control (MAC) algorithm to obtain multiuser diversity gains while preserving the optimum throughput and revenue that can be achieved in a centralized network. A fair pricing strategy is imposed to regulate the overall network performance. Fairness implies the constraint of an equal access probability. This pricing-based design motivates all selfish users to cooperate with each other.

Game-theoretic formulations for analyzing MAC protocols in the presence of selfish users that seek to maximize their own performance [5]-[10], and in particular for slotted Aloha [11]-[13], were recently proposed in the literature. The results in [11] and [13] showed that a distributed Aloha-based MAC protocol for selfish users is viable and stable. However, the throughput obtained from [11] [13] is lower than that of a centrally-controlled Aloha. Motivated by [11], in our previous work [12], we showed that by enforcing cooperation through pricing mechanisms, the throughput of the centralized slotted Aloha can be achieved in a distributed network in which selfish users access the network attempting to maximize their own utility. However, our proposed MAC schemes in [12] do not achieve multiuser diversity gains. To the best of our knowledge, none of the previously-proposed game-theoretic formulations for MAC algorithms have considered multiuser diversity as a tool to enhance the performance. In this paper, we propose a new game model which associates the channel characteristics with the transmission costs to achieve multiuser diversity gains. As opposed to our previous approach in [12], where we determined a Nash equilibrium transmission probability, here we design a Nash equilibrium threshold that takes into account statistical channel characteristics. Each user decides whether to transmit or wait by comparing his transmission cost with the Nash threshold. We consider two different optimization criteria for our MAC algorithm: throughput and revenue maximization, and we impose an equal access probability solution to enforce fairness. We further extend our model to consider multipacket reception (MPR) capabilities at the physical layer.

Both theoretical and simulation results show that the pricing model proposed in this paper leads to a distributed MAC algorithm that consumes less energy and achieves a higher spectral efficiency.

This paper is organized as follows. In Section II, a game- 
theoretic formulation for exploiting multiuser diversity is proposed. The analysis of the Nash equilibrium for the collision and MPR channel models are presented in Section III and Section IV, respectively. Simulation results are discussed in Section V and conclusions are presented in Section VI.

\section{GAME MODEL FOR EXPLOITING MULTIUSER DIVERSITY GAINS}

In this section, we present a game-theoretic model for a distributed slotted system. We assume that all users can estimate their own channel characteristics by listening to a periodically-transmitted beacon signal from the base station (see also [4]).

Each slot of the system is a one-stage game ${ }^{1}$. At the beginning of each slot, all users learn the current state of the game- - the number of users $(N)$ who currently have packets to send (active users) and their own channel characteristics. We note that, in practical systems, the number of active users can be estimated using the Pseudo-Bayesian Aloha algorithm in [14]. In this paper, we investigate the scenario where all users always have data to transmit (high-load systems), i.e. all users are active users. Each of these users performs one of the two possible actions: transmit $(T)$ or wait $(W)$. When a user transmits, its transmission can either succeed $(S)$ or fail $(F)$. The gain associated with a successful transmission is a normalized throughput of 1 , while the $\operatorname{cost}^{2}$ of transmission for user $i$ is $c_{i}$, and the network's current charge for the successful packet of user $i$ is $\mu_{i}$. If user $i$ transmits and succeeds in a given slot, then that user will receive a payoff of $\left(1-c_{i}-\mu_{i}\right)$ for that slot (throughput - cost - price paid). If the user refrains from transmission in a particular slot (waits), this will result in one slot delay for that user. We propose a generalized payoff function for the waiting cost, i.e., $v\left(c_{i}, \mu_{i}, b\right)$, which is a function of $c_{i}, \mu_{i}$ and a constant delay cost $b$. If user $i$ transmits but fails, it will incur a transmission $\operatorname{cost} c_{i}$ as well as a one-slot delay, but it will not pay for the transmission (we assume that a user is charged only for successful transmissions). Therefore, the payoff of this user in this case can be defined as $\left.-v\left(c_{i}, \mu_{i}, b\right)\right)-c_{i}$. The payoff function for user $i$ is summarized as

$$
\text { Payoff }=\left\{\begin{array}{cc}
1-c_{i}-\mu_{i}, & T \text { and } S \\
-v\left(c_{i}, \mu_{i}, b\right), & W \\
-c_{i}-v\left(c_{i}, \mu_{i}, b\right), & T \text { but } F
\end{array} .\right.
$$

In this paper, we associate this delay with the loss of the throughput the user could have achieved if it transmitted and succeeded. Thus, the payoff for this waiting user $i$ can be calculated as $-\left(1-c_{i}-\mu_{i}\right)$ (the negative of the payoff it could have achieved if successful) and $v_{i}=1-c_{i}-\mu_{i}$. Therefore, the payoff for the users who transmit but fail becomes $-1+\mu_{i}$.

\footnotetext{
${ }^{1}$ In this paper, only the one-slot payoff function is considered. Note that one slot may include many symbols depending on how fast the channels vary. Thus, this one-slot payoff function can still reflect a realistic objective function, e.g., throughput. Extensions to multi-slot payoff function will be investigated in our future work.

${ }^{2}$ The transmission cost in this paper remains constant long enough for the relative transmission frequencies to stabilize.
}

Thus, the payoff function for user $i$ is changed into

$$
\text { Payoff }=\left\{\begin{array}{cc}
1-c_{i}-\mu_{i}, & T \text { and } S \\
-\left(1-c_{i}-\mu_{i}\right), & W \\
-1+\mu_{i}, & T \text { but } F
\end{array} .\right.
$$

Note that this cost model assumes that users utilize the system resources aggressively. They value an opportunity to send since they believe that if they would have sent the packet, they would have been successful in their transmission. For this reason, the waiting cost for these users is assumed to be only associated with the payoff for the case the users successfully access the network. Note that there could be other payoff functions for the waiting users to satisfy different QoS. We have given another model in [15], which includes a constant cost penalty for waiting defined for delay-tolerant services. We have shown in [15] that similar game-theoretic analysis can be used, and also similar multi-user diversity gains can be achieved.

It is important to note that the transmission cost $c_{i}$ should be a normalized function in the range of $(0,1)$ and is related to the transmission energy cost. In wireless systems, channels change from one slot to another. The higher the channel power gain, the lower the transmission power (hence transmission cost) required to achieve the same spectral efficiency. Thus, the transmission cost should be defined as a monotonicallydecreasing function of the channel power gains.

\section{EQUILIBRIA FOR THE COLLISION MODEL}

In this section, we consider the classical collision model, for which a transmission is considered to be successful only when a single user transmits. Let $s_{i}$ be the strategy taken by user $i$ and $s_{i} \in\{T, W\}$. Thus, the payoff function of user $i$ in (2) can be written as a function of the strategies of all users, i.e.,

Payoff of user i

$$
=\left\{\begin{array}{cc}
1-c_{i}-\mu_{i}, & s_{i}=T, s_{j}=W \text { for all } j \neq i \\
-\left(1-c_{i}-\mu_{i}\right), & s_{i}=W, s_{j \neq i}=\{T, W\} \\
-1+\mu_{i}, & s_{i}=T, s_{j}=T \text { for at least one } j \neq i
\end{array}\right.
$$

In this paper, we consider a fair game model where all users access the system with the same probability $p$. Then, the expected payoff user $i$ gets from selecting action $T$ is

$$
\begin{aligned}
& U_{i}(T)=U_{i}\left(s_{i}=T, s_{-i}\right) \\
& =\left(-1+\mu_{i}\right)\left[1-(1-p)^{N-1}\right]+\left(1-c_{i}-\mu_{i}\right)(1-p)^{N-1},
\end{aligned}
$$

where $s_{-i}=s_{j \neq i}$. The expected payoff user $i$ gets with action $W$ is

$$
U_{i}(W)=U_{i}\left(s_{i}=W, s_{-i}\right)=-\left(1-c_{i}-\mu_{i}\right) .
$$

In [12], a nondegenerate mixed strategy equilibrium from (4) and (5) is obtained using the indifference principle. However, this mixed strategy equilibrium cannot achieve multiuser diversity gains. In this paper, our goal is to optimize the system performance by appropriately selecting the price $\mu_{i}$, according to the transmission costs $\left\{c_{i}, i=1, \cdots, N\right\}$ of the users, so that each user can achieve its maximum expected payoff by taking appropriate actions: $T$ or $W$. If the expected payoff 
of $T$ is larger than $W$, then the user transmits, otherwise, he waits. Then, the Nash equilibrium can be defined as follows:

A Nash equilibrium strategy for the set of $N$ players will be a profile of strategies $\left(s_{1}^{*}, \ldots, s_{N}^{*}\right)$, where $s_{i}^{*} \in\{T, W\}$, $i=1,2, \ldots, N$ such that for any player $i, \forall s_{i}^{\prime} \in\{T, W\}$, $s_{i}^{\prime} \neq s_{i}^{*}, U_{i}\left(s_{i}^{*}, s_{-i}^{*}\right) \geq U_{i}\left(s_{i}^{\prime}, s_{-i}^{*}\right)$, where $s_{-i}^{*}=s_{j \neq i}^{*}, j=$ $1,2, \ldots, N$.

Note that $U_{i}($.$) represents the expected payoff defined in$ (4) and (5), and accounts for the mixed equilibrium strategies of the other players. This equilibrium changes from one slot to another as the transmission costs change.

To maximize its own expected payoff, a user will take an action $T$ when $U_{i}(T) \geq U_{i}(W)$, otherwise, it will wait. From (4) and (5), we can obtain

$$
U_{i}(T) \geq U_{i}(W) \Rightarrow c_{i} \leq \frac{2\left(1-\mu_{i}\right)(1-p)^{N-1}}{1+(1-p)^{N-1}} .
$$

We define

$$
\tau_{i}=\frac{2\left(1-\mu_{i}\right)(1-p)^{N-1}}{1+(1-p)^{N-1}},
$$

where $\tau_{i}$ is called the equilibrium threshold, and we can obtain

$$
c_{i} \leq \tau_{i}
$$

and

$$
\mu_{i}=1-\frac{\tau_{i}+\tau_{i}(1-p)^{N-1}}{2(1-p)^{N-1}} .
$$

Note that each user estimates his channel power gain through a periodically-transmitted beacon signal and learns the best strategy periodically by comparing the transmission cost $c_{i}$ (which is a function of channel power gain) with the equilibrium threshold $\tau_{i}$. Once $\tau_{i}$ is obtained, each user can decide whether it should transmit or not. Consequently, the problem of finding a Nash equilibrium strategy is reduced to the problem of finding an equilibrium threshold. Users whose transmission costs are smaller than their corresponding equilibrium thresholds will transmit their packets during this time slot.

Now, let us analyze the relationship among $\tau_{i}, p$ and $\mu_{i}$. Since $p$ is the probability that a user transmits, we have

$$
p=P\left\{U_{i}(T) \geq U_{i}(W)\right\}=P\left\{c_{i} \leq \tau_{i}\right\} .
$$

Let $F_{c_{i}}($.$) denote the cumulative probability distribution$ function (CDF) of $c_{i}$. Then

$$
F_{c_{i}}\left(\tau_{i}\right)=P\left\{c_{i}<\tau_{i}\right\}
$$

From (10) and (9), we can obtain

$$
\begin{gathered}
p=F_{c_{i}}\left(\tau_{i}\right), \\
\tau_{i}=F_{c_{i}}^{-1}(p),
\end{gathered}
$$

and

$$
\mu_{i}=1-\frac{F_{c_{i}}^{-1}(p)+F_{c_{i}}^{-1}(p)(1-p)^{N-1}}{2(1-p)^{N-1}} .
$$

\section{Case Study:}

Let $G_{i}$ denote the channel power gain of the $i$-th user and $F_{i}($.$) denote the CDF of G_{i}$. We define the transmission cost to be $c_{i}=1-F_{i}\left(G_{i}\right)$. This definition preserves the required properties for $c_{i}$, i.e., normalization and monotonic decrease with the increased channel power gains.

Note that there may be some other definitions of $c_{i}$ which satisfy the required properties. Our definition simplifies the theoretical analysis. Substituting $c_{i}=1-F_{i}\left(G_{i}\right)$ into (11), we obtain

$$
\begin{aligned}
& F_{c_{i}}\left(\tau_{i}\right)=P\left\{1-F_{i}\left(G_{i}\right)<\tau_{i}\right\}=P\left\{G_{i}>F_{i}^{-1}\left(1-\tau_{i}\right)\right\} \\
& =1-F_{i}\left\{F_{i}^{-1}\left(1-\tau_{i}\right)\right\}=\tau_{i} .
\end{aligned}
$$

From (12), we obtain

$$
\tau_{i}=p, \quad i=1,2, \ldots, N .
$$

Substituting (16) into (9) gives

$$
\mu_{i}=1-\frac{p+p(1-p)^{N-1}}{2(1-p)^{N-1}} \triangleq \mu, \quad i=1,2, \ldots, N .
$$

It is interesting to note that the equilibrium threshold is the same as the transmission probability $p$, which is decided by the price $\mu_{i}$ (from (17)) charged by the network. Note that $p$ is the same for all the users. Since $\mu$ is a decreasing function of $p$, to obtain a larger $p$, the network needs to reduce pricing. In turn, the transmission probability will influence the achievable network throughput, and in combination with the pricing scheme, the revenue of the network provider.

Since the classical collision channel model is considered in this section, the transmission is successful only if there is exactly one user whose transmission cost is smaller than the equilibrium threshold. Since the successful user is the only one attempting transmission, according to our Nash equilibrium threshold design, it must be the user with the lowest transmission cost.

- In homogeneous systems, all users have the same statistical channel characteristics so that $\left\{F_{i}\right\}$ is the same for all users. The lowest transmission cost is thus incurred at the highest channel power gain so that the successful user must be the one with the largest channel power gain and thus the multiuser diversity gain is achieved.

- In heterogeneous systems, different users have different statistical channel characteristics. For two types of users with different statistical channel characteristics, a lower transmission cost is not necessarily caused by a higher channel power gain. Therefore, the successful user must be the one with the lowest transmission cost but may not necessarily be the one with the highest channel power gain. However, since the user with the lowest transmission cost must have the largest channel power gain among all the users having the same type of statistical channel characteristics, the multiuser diversity gain can still be achieved among the users of the same type.

In the following, we will optimize the pricing scheme, given the two above-mentioned criteria: throughput and revenue.

\section{A. Throughput Optimization}

This subsection describes how the network adjusts pricing to achieve the maximum throughput. It is well known that the network throughput $S$ of a slotted Aloha system is

$$
S=N p(1-p)^{N-1}
$$


and the maximum throughput is achieved when $p=1 / N$ [14]. To obtain the same maximum throughput, we set the transmission probability to $p=1 / N$. Therefore, from (14), the optimal pricing to maximize the throughput is

$$
\mu_{i}=1-\frac{F_{c_{i}}^{-1}\left(\frac{1}{N}\right)+F_{c_{i}}^{-1}\left(\frac{1}{N}\right)\left(1-\frac{1}{N}\right)^{N-1}}{2\left(1-\frac{1}{N}\right)^{N-1}},
$$

and the equilibrium threshold is

$$
\tau_{i}=F_{c_{i}}^{-1}\left(\frac{1}{N}\right)
$$

Case Study $c_{i}=1-F_{i}\left(G_{i}\right)$ :

From (16) and (17), we obtain that

$$
\mu=\mu_{i}=1-\frac{\frac{1}{N}+\frac{1}{N}\left(1-\frac{1}{N}\right)^{N-1}}{2\left(1-\frac{1}{N}\right)^{N-1}}, \quad i=1,2, \ldots, N
$$

and the equilibrium threshold is

$$
\tau_{i}=p=\frac{1}{N}, \quad i=1,2, \ldots, N .
$$

At first glance, it may seem that pricing does not have any effect on the performance since the equilibrium threshold is equal to $\frac{1}{N}$. However, without pricing, i.e. $\mu_{i}=0$, from (7) we have

$$
p=\tau_{i}=\frac{2(1-p)^{N-1}}{1+(1-p)^{N-1}} .
$$

The numerical solutions of (23) for different numbers of users, are given in Fig. 1. a, where it can be seen that the thresholds obtained without pricing from (23) are different from our proposed pricing solutions which maximize the throughput ( $\tau=\frac{1}{N}$, the curve denoted as 'Throughput optimization'). Thus, by introducing pricing, the network can regulate the overall network behavior.

The pricing $\mu$ is monotonically increasing with the number of users as shown in Fig. 1. b (the curve denoted as 'Throughput optimization'). This result is intuitively appealing, since the network should reduce its charging price to encourage users to transmit when there are only few users in the system. When $N=1$, there is only one user in the system. In this case, $\mu=0$ and $\tau=1$ so that this user is always transmitting data. For the extreme case of $N=\infty, \mu=1$ and $\tau=0$ and no user will try to transmit since there will always be collisions.

\section{B. Revenue Optimization}

In the previous subsection, the network decides the price aiming to maximize the throughput of the whole network. In this subsection, we consider a more realistic objective for the service provider which is to maximize revenue. Assume that the network only charges for successful transmissions. To achieve fairness, the transmission probabilities for all users are enforced to be the same and hence the revenue for the network is given by

$$
R=\sum_{i=1}^{N} \mu_{i} p(1-p)^{N-1}
$$

Substituting $\mu_{i}$ given in (14) into (24), we obtain

$$
R=\sum_{i=1}^{N}\left(1-\frac{F_{c_{i}}^{-1}(p)+F_{c_{i}}^{-1}(p)(1-p)^{N-1}}{2(1-p)^{N-1}}\right) p(1-p)^{N-1} .
$$

The optimum $p$ which maximizes $R$ can be obtained by setting the derivative of (25) to zero.

Case Study $c_{i}=1-F_{i}\left(G_{i}\right)$ :

Substituting (17) into (24), we obtain

$$
R=\left(1-\frac{p+p(1-p)^{N-1}}{2(1-p)^{N-1}}\right) N p(1-p)^{N-1} .
$$

The closed-form solution for the optimum $p$ is analytically intractable. However, several optimization software packages can be used to compute the numerical solutions, such as the fminsearch function in MATLAB. After we obtain the optimum solution, the price $\mu$ can be obtained from (17) and the equilibrium threshold can be obtained from (16).

Next, we will investigate the relationship between these two criteria. It is well-known from the centrally-controlled slotted Aloha protocol that $S=N p(1-p)^{N-1}$ is a strictly-increasing function over $p$ when $p<\frac{1}{N}$, and a strictly-decreasing function when $p>\frac{1}{N}$ [14]. The maximum $S$ is achieved at $p=\frac{1}{N}$. From (17), it can be seen that $\mu$ is a decreasing function of $p$. Therefore, the optimum $p$ maximizing $R$ must be no larger than the optimum $p$ maximizing $S$ since $R=\mu S$. Then, the optimum pricing for the revenue optimization should be no smaller than the optimum pricing for the throughput optimization. We can see these facts from the thresholds and pricing comparisons in Fig. 1. a and Fig. 1. b, respectively.

Another observation we notice from Fig. 1 . b is that $\mu<1$. Thus, when a user transmits but fails, the expected payoff becomes negative, i.e. $-1+\mu_{i}<0$. However, the expected payoff of waiting $\left(-\left(1-c_{i}-\mu_{i}\right)\right)$ may be positive depending on the transmission cost since the larger the transmission cost, the larger the expected payoff of waiting. In fact, if the transmission cost is high, e.g., $c_{i}>1-\mu_{i}$, the expected payoff of waiting is positive and the payoffs for $T$ and $S$ can even become negative. Consequently, this user would like to wait rather than transmit and the resulting average expected payoff is still positive as shown in the simulation section. This observation reinforces our explanation regarding the possibility of achieving multiuser diversity.

\section{EQUILIBRIA FOR THE MULTIPACKET RECEPTION} MODEL

In the previous section, we analyzed the fundamental collision model, for which two simultaneously-transmitted packets always collide and are assumed lost. However, enhancements at the physical layer (e.g., beamforming, spatial diversity, multiuser detection, etc.) result in multipacket reception (MPR) capabilities, i.e., more packets may be successfully received simultaneously. In this section, we use the game model presented in Section II to analyze the MPR channels. We have adopted the analysis model in [13] and [16]. The channel is 


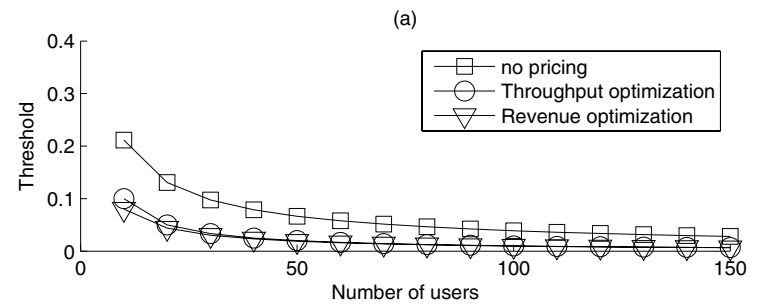

(b)

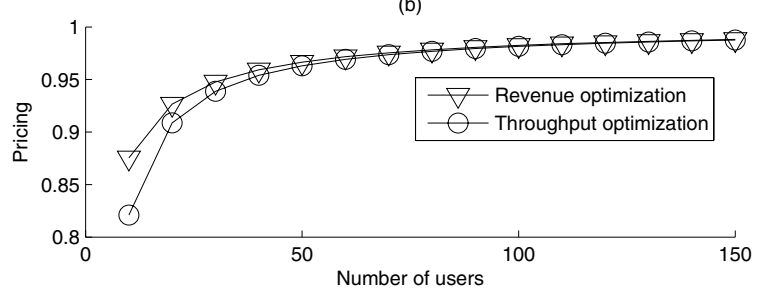

Fig. 1. Threshold and pricing comparison for the collision model

described by an MPR matrix

$$
B=\left[\begin{array}{ccccccc}
\rho_{10} & \rho_{11} & 0 & 0 & \cdots & 0 & \cdots \\
\rho_{20} & \rho_{21} & \rho_{22} & 0 & \cdots & 0 & \cdots \\
\cdots & \cdots & \cdots & \cdots & \cdots & \cdots & \cdots \\
\rho_{n 0} & \rho_{n 1} & \rho_{n 2} & \cdots & \rho_{n n} & 0 & \cdots \\
\cdots & \cdots & \cdots & \cdots & \cdots & \cdots & \cdots
\end{array}\right],
$$

where $\rho_{n l}$ denotes the probability that $l$ packets are successfully received in a slot where $n$ packets are transmitted. Similar to [13], the expected number of successes in a total of $n$ transmissions is given by

$$
r_{n}=\sum_{l=0}^{n} l \rho_{n l}
$$

Let $b_{p}(n, k)=\left(\begin{array}{c}n \\ k\end{array}\right) p^{k}(1-p)^{n-k}$ be the probability of $k$ packets transmitted out of $n$ packets, where $p$ is the equilibrium transmission probability for all the players. Similar to the Aloha game for the collision model, a Nash equilibrium threshold exists for this finite strategy form game. We derive the equilibrium strategy in a similar fashion as before.

The expected payoff for transmission is

$$
\begin{aligned}
& U_{i}(T)=\left(1-c_{i}-\mu_{i}\right)\left[\sum_{k=1}^{N} b_{p}(N-1, k-1) \frac{r_{k}}{k}\right] \\
& +\left(-1+\mu_{i}\right)\left[1-\sum_{k=1}^{N} b_{p}(N-1, k-1) \frac{r_{k}}{k}\right] .
\end{aligned}
$$

The expected payoff for waiting is

$$
U_{i}(W)=-\left(1-c_{i}-\mu_{i}\right) .
$$

Similar to the collision model, if $U_{i}(T) \geq U_{i}(W)$, the player will transmit, otherwise, it will wait. Since

$$
\begin{aligned}
U_{i}(T) & \geq U_{i}(W) \Rightarrow c_{i} \\
& \leq \frac{2\left(1-\mu_{i}\right)\left(\sum_{k=1}^{N} b_{p}(N-1, k-1) \frac{r_{k}}{k}\right)}{1+\sum_{k=1}^{N} b_{p}(N-1, k-1) \frac{r_{k}}{k}},
\end{aligned}
$$

the Nash equilibrium threshold $\tau_{i}$ is defined as

$$
\tau_{i}=\frac{2\left(1-\mu_{i}\right)\left(\sum_{k=1}^{N} b_{p}(N-1, k-1) \frac{r_{k}}{k}\right)}{1+\sum_{k=1}^{N} b_{p}(N-1, k-1) \frac{r_{k}}{k}} .
$$

It can be easily seen that the derivation of the relationship among $\tau_{i}$ and $p$ in Section III is also true for the multipacket model. Thus, substituting (13) into (32), we get

$$
\mu_{i}=1-\frac{F_{c_{i}}^{-1}(p)+F_{c_{i}}^{-1}(p) \sum_{k=1}^{N} b_{p}(N-1, k-1) \frac{r_{k}}{k}}{2 \sum_{k=1}^{N} b_{p}(N-1, k-1) \frac{r_{k}}{k}}
$$

Let

$$
\alpha=\sum_{k=1}^{N} b_{p}(N-1, k-1) \frac{r_{k}}{k} .
$$

When $N$ is very large and $p$ is very small, let $\gamma=N p$ and use the Poisson approximation ${ }^{3}$ to (34) to obtain

$$
\alpha \cong \sum_{k=1}^{N} \frac{e^{-\hat{\gamma}} \hat{\gamma}^{k-1} r_{k}}{k !}
$$

Thus,

$\mu_{i}=1-\frac{F_{c_{i}}^{-1}(p)+F_{c_{i}}^{-1}(p) \alpha}{2 \alpha}=1-\frac{F_{c_{i}}^{-1}\left(\frac{\gamma}{N}\right)+F_{c_{i}}^{-1}\left(\frac{\gamma}{N}\right) \alpha}{2 \alpha}$.

Let $\hat{\gamma}$ denote the solution of (36). According to [13], the system is stable if

$$
\lambda<e^{-\hat{\gamma}} \sum_{k=1}^{\infty} \frac{\hat{\gamma}^{k}}{k !} r_{k},
$$

where $\lambda$ is the arrival rate of the selfish users. For the slotted Aloha with MPR model, the authors in [16] showed that stability is achieved if

$$
\lambda<\sup _{x \geq 0} e^{-x} \sum_{k=1}^{\infty} \frac{x^{k}}{k !} r_{k} .
$$

If there is a value of $x>0$ achieving this supremum, then there exists a $\hat{\gamma}=x$, and thus an equilibrium threshold $\tau_{i}=$ $F_{c_{i}}^{-1}\left(\frac{x}{N}\right)$ that achieves the maximum throughput. Viewing (36) as an equation in $\gamma$, we find that different pricing $\mu_{i}$ results in different $\hat{\gamma}$. Therefore, the network operator can adjust the pricing $\mu_{i}=1-\frac{F_{c_{i}}^{-1}\left(\frac{x}{N}\right)+F_{c_{i}}^{-1}\left(\frac{x}{N}\right) \alpha}{2 \alpha}$ to make $\hat{\gamma}=x$.

Similar to the analysis of the collision model, we know from (31) that users with lower transmission costs will transmit so that the multiuser diversity gains are achieved. Fairness among all users in heterogeneous systems can be guaranteed by the enforced equal-access probability.

In the following, we use the capture model in [16] to investigate the effect of pricing on the random access protocol to obtain multiuser diversity gains. The MPR matrix can be

\footnotetext{
${ }^{3}$ Since we use the Poisson approximation to the binomial distribution, the obtained results are valid for large $N$, i.e., $N>10$.
} 
redefined as

$$
B=\left[\begin{array}{ccccccc}
0 & 1 & 0 & 0 & \cdots & 0 & \cdots \\
1-1 / \beta^{2} & 1 / \beta^{2} & 0 & 0 & \cdots & 0 & \cdots \\
\cdots & \cdots & \cdots & \cdots & \cdots & \cdots & \cdots \\
1-1 / \beta^{2} & 1 / \beta^{2} & 0 & \cdots & 0 & 0 & \cdots \\
\cdots & \cdots & \cdots & \cdots & \cdots & \cdots & \cdots
\end{array}\right]_{(39)}
$$

so that $r_{1}=1$ and $r_{k}=1 / \beta^{2}$ for $k \neq 1$ according to (28) where $\beta$ is a measure of the packet capture capability of the system such that smaller $\beta$ denotes a stronger capture capability. By substituting $r_{1}=1$ and $r_{k}=1 / \beta^{2}$ for $k \neq 1$ into (35) and assuming $N$ is very large we get

$$
\alpha \cong \sum_{k=1}^{\infty} \frac{e^{-\hat{\gamma}} \hat{\gamma}^{k-1} r_{k}}{k !}=\frac{e^{-\hat{\gamma}}\left(\beta^{2} \hat{\gamma}-\hat{\gamma}-1\right)+1}{\beta^{2} \hat{\gamma}},
$$

In what follows, we optimize the network pricing strategy according to the same two criteria previously discussed: throughput and revenue maximization.

\section{A. Throughput Optimization}

In this subsection, we use throughput optimization as a metric. From (38), we know that the optimal throughput is

$$
s=e^{-\hat{\gamma}} \sum_{k=1}^{\infty} \frac{\hat{\gamma}^{k}}{k !} r_{k} .
$$

Substituting (35) into (41), we obtain

$$
s=\sum_{k=1}^{\infty} \frac{e^{-\hat{\gamma}} \hat{\gamma}^{k} r_{k}}{k !}=\alpha \hat{\gamma} .
$$

Since the maximum throughput achieved with the optimal decentralized control algorithm is $1 / \beta^{2}+(1-$ $\left.1 / \beta^{2}\right) e^{\left(-\beta^{2} /\left(\beta^{2}-1\right)\right)}[17]$, the optimum $\hat{\gamma}$ satisfies

$$
\alpha \hat{\gamma}=1 / \beta^{2}+\left(1-1 / \beta^{2}\right) e^{-\beta^{2} /\left(\beta^{2}-1\right)} .
$$

From (43) and (40), we obtain

$$
e^{-\hat{\gamma}}\left(\beta^{2} \hat{\gamma}-1-\hat{\gamma}\right)=\left(\beta^{2}-1\right) e^{-\beta^{2} /\left(\beta^{2}-1\right)} .
$$

It can be seen that $\hat{\gamma}=\frac{\beta^{2}}{\beta^{2}-1}$ is the solution of (44). Therefore,

$$
p=\frac{\hat{\gamma}}{N}=\frac{\beta^{2}}{N\left(\beta^{2}-1\right)}
$$

and

$$
\alpha=\frac{\beta^{2}-1}{\beta^{4}}+\frac{\left(\beta^{2}-1\right)^{2}}{\beta^{4}} e^{-\frac{\beta^{2}}{\beta^{2}-1}} .
$$

Then, from (13), we obtain the equilibrium threshold

$$
\tau_{i}=F_{c_{i}}^{-1}(p)=F_{c_{i}}^{-1}\left(\frac{\beta^{2}}{N\left(\beta^{2}-1\right)}\right),
$$

and the pricing policy can be obtained from (36).

Case Study $c_{i}=1-F_{i}\left(G_{i}\right)$ :

From (45), (16) and (36), we obtain that

$$
\tau_{i}=p=\frac{\beta^{2}}{N\left(\beta^{2}-1\right)}, \quad i=1,2, \ldots, N
$$

and

$$
\begin{aligned}
& \mu_{i}=1-\frac{\tau_{i}+\tau_{i} \alpha}{2 \alpha} \\
& =1-\frac{\beta^{2}\left[\beta^{4}+\left(\beta^{2}-1\right)+\left(\beta^{2}-1\right)^{2} e^{-\frac{\beta^{2}}{\beta^{2}-1}}\right]}{2 N\left(\beta^{2}-1\right)\left[\left(\beta^{2}-1\right)+\left(\beta^{2}-1\right)^{2} e^{-\frac{\beta^{2}}{\beta^{2}-1}}\right]} .
\end{aligned}
$$

The equilibrium thresholds and pricing under different values of the capture parameter $\beta$ can be seen from Fig. 2 and Fig. 3. Similar to the collision model, the equilibrium threshold monotonically decreases with the number of users while the pricing monotonically increases with the number of users. When $\beta$ is small, which means the system has stronger capture capabilities, a reduced pricing is designed by the network so as to encourage users to transmit. Interestingly, we find that when the number of users is very small, i.e., in a very lowload system, the service provider even has to pay the user (i.e., the pricing is negative) to make the system operate at the maximum throughput. Evidently, the negative pricing would not happen in practice. Only positive pricing makes sense from the service provider point of view (even if the system operates at suboptimal throughput). However, we see a price rebate phenomenon in real systems, when the network provider tries to attract more customers to join the network.

\section{B. Revenue Optimization}

We now analyze how to select a pricing policy that maximizes revenue for the service provider. For $N$ users in the system, the amount of service provided by the network to each user is $p \sum_{k=1}^{N}\left[b_{p}(N-1, k-1) \frac{r_{k}}{k}\right]$. Therefore, the revenue of the network is

$$
R=\sum_{i=1}^{N} \mu_{i} p\left(\sum_{k=1}^{N}\left[b_{p}(N-1, k-1) \frac{r_{k}}{k}\right]\right) .
$$

When $N$ is very large and $p$ is very small, the network revenue can be determined as

$$
R=\sum_{i=1}^{N} \mu_{i} p \alpha,
$$

where $p=\hat{\gamma} / N, \alpha$ and $\mu_{i}$ are given in (40) and (36), respectively. Thus, $\mathrm{R}$ becomes a function of $\hat{\gamma}$ and the optimum $\hat{\gamma}$ can be obtained by setting the derivative of $R$ to zero. Correspondingly, the equilibrium thresholds and the pricing can be obtained from $\hat{\gamma}$.

Case Study $c_{i}=1-F_{i}\left(G_{i}\right)$ :

In this case, (36) becomes

$$
\mu_{i}=1-\frac{\frac{\hat{\gamma}}{N}}{2}-\frac{\frac{\hat{\gamma}}{N}}{2 \alpha}
$$

and (51) becomes

$$
R=\frac{e^{-\hat{\gamma}}\left(\beta^{2} \hat{\gamma}-\hat{\gamma}-1\right)(2 N-\hat{\gamma})+2 N-\hat{\gamma}-\hat{\gamma}^{2} \beta^{2}}{2 N \beta^{2}} .
$$

The optimum $\hat{\gamma}$ can be obtained numerically. The corresponding equilibrium thresholds and the pricing are shown in Fig. 2 and Fig. 3. It can be seen that the thresholds decrease with the number of users while pricing increases 
with the number of users. Similar to the collision model, the thresholds of the revenue optimization are smaller than the thresholds of the throughput optimization while the pricing of the revenue optimization is larger than the pricing of the throughput optimization. Different from the throughout optimization where the thresholds and pricing vary significantly with $\beta$, the thresholds and pricing vary only slightly with $\beta$ for the revenue optimization scenario.

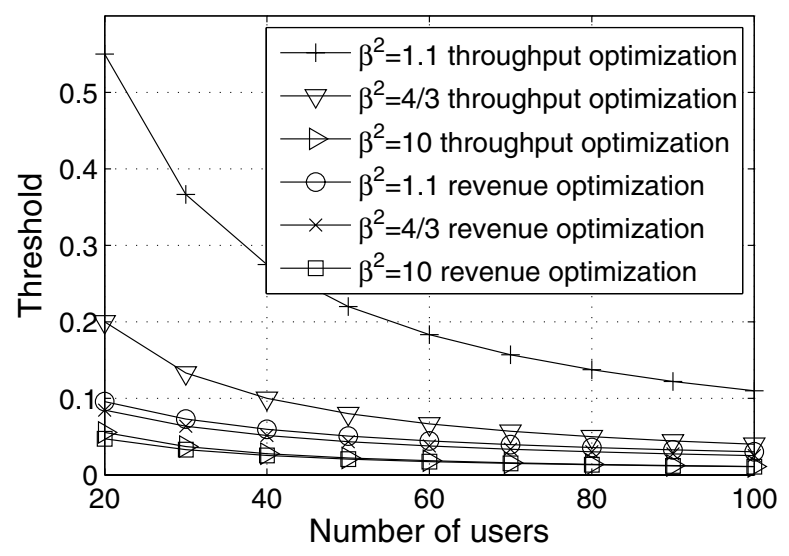

Fig. 2. Threshold comparison for the MPR model

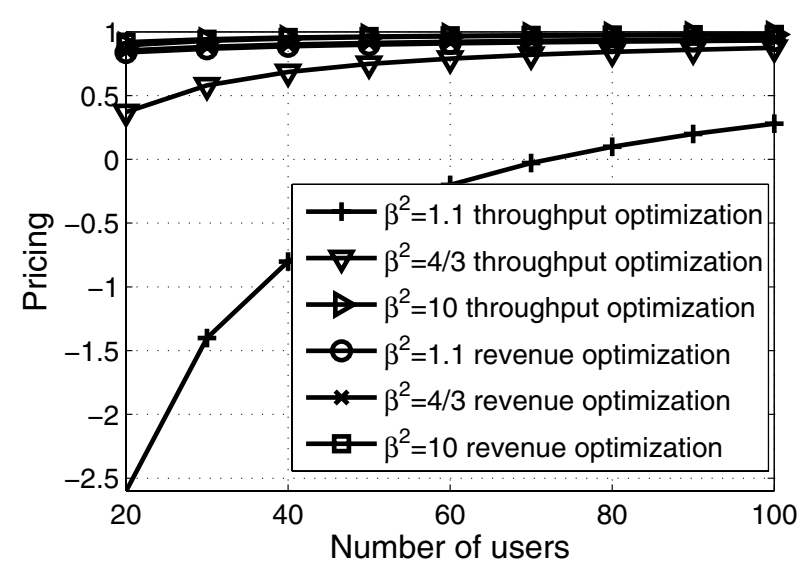

Fig. 3. Pricing comparison for the MPR model

\section{Numerical and Simulation Results}

In the previous sections, we described the game model for our MAC scheme and derived the Nash equilibrium thresholds for both the collision and MPR models. In this section, both simulation and numerical results are provided. The simulation results are obtained from 100,000 independent slots and we assume a Bit-Error-Rate (BER) requirement of BER $=10^{-5}$ and a corresponding Signal-to-Noise Ratio (SNR) requirement of $\mathrm{SNR}=15 \mathrm{~dB}$. In this section, we investigate the performance of the special case that $c_{i}=1-F_{i}\left(G_{i}\right)$. To show the multiuser diversity gains in the simulation, we will consider two performance criteria: spectral efficiency and energy consumption, which are defined as follows.

\section{1) Spectral efficiency}

The metric of spectral efficiency is used to measure how many bits a user can transmit for a given bandwidth. Here, a continuous-rate adaptive modulation strategy is used by exploiting the instantaneous channel power gains, and the spectral efficiency is calculated as [18]

$$
r=\log _{2}\left(1+\frac{\Gamma P G_{i}}{\sigma_{i}^{2}}\right),
$$

where $P$ is the transmission power, $\sigma_{i}^{2}$ is the noise power and $\Gamma$ is the SNR gap given by

$$
\Gamma=-\frac{1.5}{\ln (5 \mathrm{BER})} \text {. }
$$

Note that to show the effects of the channel gains on the number of bits transmitted, we consider a fixed power $P=1$ in the evaluation of spectral efficiency.

2) Energy consumption

The metric of energy consumption is used to evaluate how much energy is consumed by various users to achieve a given data rate with the same pre-specified BER requirement. Note that the users who transmit but fail will still consume the same amount of energy as if they transmitted and succeeded as defined in the game model in Section II. The difference is that they do not need to pay anything if their packets are not successfully received. From (54), we can calculate the power $P$ to achieve data rate $r$ as

$$
P=\frac{\left(2^{r}-1\right) \sigma_{i}^{2}}{\Gamma G_{i}} .
$$

In this simulation, we use the normalized data rate $r=1$ to evaluate the energy consumption and $\Gamma$ is defined as in (55). It can be seen from (56) that the higher the channel power gain $\left(G_{i}\right)$ of the user, the smaller its power consumption is.

To simplify the notations for illustration purposes, our proposed game scheme in this paper is denoted as COOPMUD-ALOHA, where MUD is for multiuser diversity. We run simulations and present theoretical results to illustrate the performance of our new proposed scheme, and compare the performance results with the following two benchmark algorithms

- Conventional slotted Aloha (CONV-ALOHA) [14] [17]: For the collision model, each user transmits with a probability $\frac{1}{N}$ ( [14]) irrespective of his channel characteristics. For the MPR model, the optimal transmit probability in [17] is adopted, i.e., $p=\frac{\beta^{2}}{N\left(\beta^{2}-1\right)}$.

- MAC scheme proposed in our previous work [12] (COOP-ALOHA): To make a fair comparison with the proposed scheme in this paper, we redefine $c_{i}$ from [12] to be the same as the transmission cost in this paper, i.e., $c_{i}=1-F_{i}\left(G_{i}\right)$ (note that in [12], we define $c_{i}$ in terms of the fraction of the total battery energy level). This redefinition of $c_{i}$ does not affect the computed Nash equilibrium in [12] for the collision model. However, for the MPR model, the derivations in [12] do not hold anymore since for the revenue optimization given in [12], we assume that $\mu_{i}$ and $c_{i}$ are constant while with the 
redefinition, $c_{i}$ is changing with channel power gain. Consequently, we only compare our scheme with COOP-

ALOHA for the collision model.

In the following two subsections, we present simulation and numerical results for both the collision and the MPR models.

\section{A. Collision Model}

1) System Model: We assume that the channels between users and the access point are characterized by independent fading. The lowpass-equivalent channel gains $\left\{h_{i}, i=\right.$ $1,2, \ldots N\}$ between an arbitrary user $i$ and the access point are assumed to be independent zero-mean unit-variance circularlysymmetric complex Gaussian random variables. Consequently, the channel power gains $G_{i}=\left|h_{i}\right|^{2}(i=1,2, \ldots, N)$ have exponential distribution with unit mean [19], i.e,

$$
F_{i}\left(G_{i}\right)=\left(1-e^{-G_{i}}\right) u\left(G_{i}\right),
$$

where $u\left(G_{i}\right)$ is the unit step function. Correspondingly, the transmission cost of user $i$ is

$$
c_{i}=1-F_{i}\left(G_{i}\right)=e^{-G_{i}} u\left(G_{i}\right) .
$$

2) Numerical and Simulation Results: Figs. 4, 5, 6 and 7 give the numerical and simulation results for the collision model. From Figs. 4 and 5, we can see that our simulation results match very well with our theoretical results which are calculated from (18) and (24). COOP-MUD-ALOHA based on throughput optimization achieves almost the same performance as COOP-MUD-ALOHA based on revenue optimization. It is interesting to note that both COOP-ALOHA and COOP-MUD-ALOHA achieve the same throughput performance for the case of throughput optimization. However, if revenue is optimized, the revenue obtained for COOP-ALOHA is much less than that obtained by the COOP-MUD-ALOHA scheme.

The reason for the above observations is that in COOPALOHA, the price changes with the transmission costs to encourage equal-access probability even when the transmission cost is high. However, in COOP-MUD-ALOHA, the price remains the same and it is usually higher than the one for COOP-ALOHA. In COOP-ALOHA, when the transmission cost of a user is larger, the network tends to reduce the price of that user to impose the equal-access probability. In contrast, in COOP-MUD-ALOHA, the successful user is always the user with the smallest transmission cost. Therefore, most of the prices in COOP-MUD-ALOHA are higher than the prices in COOP-ALOHA.

Fig. 6 compares the spectral efficiencies of different schemes. COOP-MUD-ALOHA again achieves much higher spectral efficiency than other schemes for both cases of throughput optimization and revenue optimization. Fig. 7 compares the energy consumption for different MAC schemes. It can be seen that COOP-MUD-ALOHA consumes much less energy compared to both CONV-ALOHA and COOPALOHA.

From the presented simulation results, we can see that our proposed COOP-MUD-ALOHA achieves much higher multiuser diversity gains compared with the other schemes (Figs. 6 and 7), without sacrificing throughput or revenue
(Figs. 4 and 5). In addition, throughput optimization based COOP-MUD-ALOHA has almost the same performance as the revenue optimization based COOP-MUD-ALOHA. However, for COOP-ALOHA in [12], the network must tradeoff throughput optimization and revenue optimization as shown in Figs. 4 and 5. The average expected payoff is shown in Fig. 8. It is clear that the average expected payoff is positive because of the pricing policy.

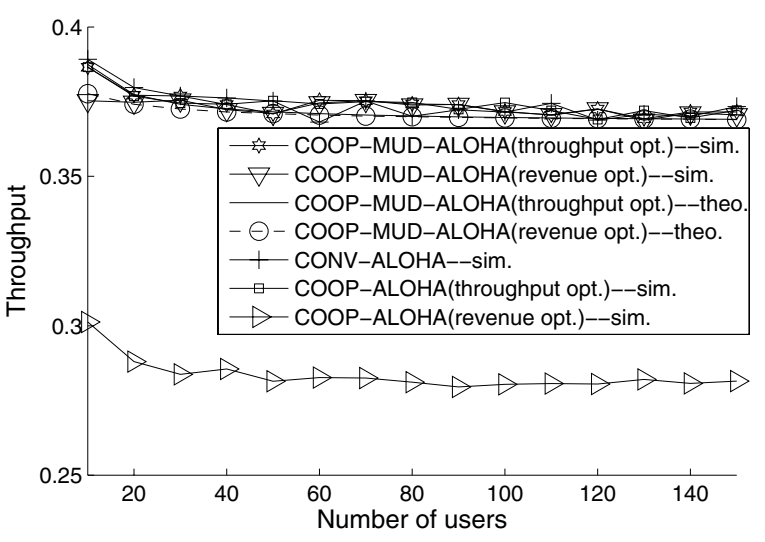

Fig. 4. Throughput comparison for the collision model

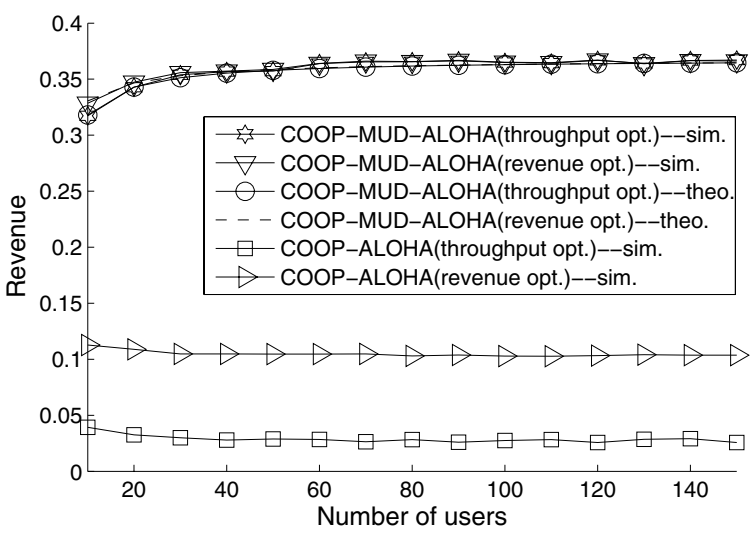

Fig. 5. Revenue comparison for the collision model

\section{B. Multipacket Reception Model}

1) System Model: In this section, we present our simulation results based on the MPR model in Section III. We assume that there are $N$ users independently and uniformly distributed in a circle of radius $\mathrm{D}$ whose positions are independent from slot to slot. Users transmit packets to an access point at the center of the network. Without loss of generality, we assume that Users 1 and 2 have the strongest and the second strongest received powers, respectively, among all the users whose packets are involved in a collision. As in [16], the strongest packet is correctly received iff $\frac{G_{1}}{G_{2}}>K(K$ being a system-dependent constant), and all the other packets involved in the collision are not received successfully. Let $d_{i}$ denote the distance between user $i$ and the access point. Moreover, assume that the received 


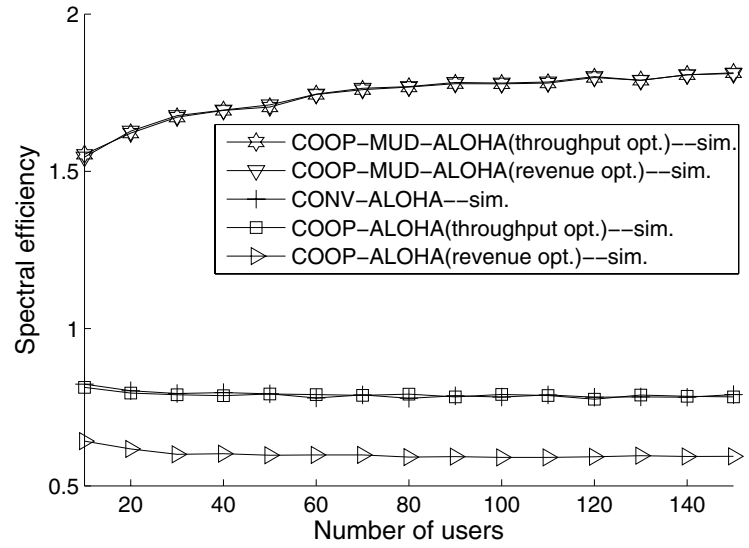

Fig. 6. Spectral efficiency comparison for the collision model

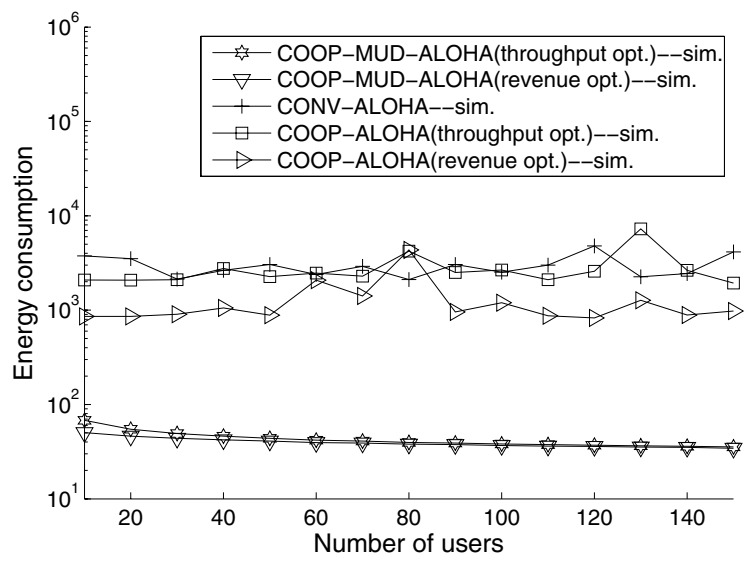

Fig. 7. Energy consumption comparison for the collision model

power of a packet only depends on the distance $d_{i}$

$$
G_{i}=\frac{A}{d_{i}^{\alpha}}, \alpha \geq 2
$$

where we assume $A=1$ and $\alpha=4$ in our simulation. Then, there will be capture iff

$$
d_{2}>\beta d_{1},
$$

where $\beta=K^{\frac{1}{\alpha}}$ is the capture parameter, and $d_{1}, d_{2}$ are the distances of the closest and the next to closest senders from the receiver, respectively. In our simulation, we set $\beta^{2}=\frac{4}{3}$. The probability density function (pdf) of $d_{i}$ is

$$
f\left(d_{i}\right)=\left\{\begin{array}{cc}
\frac{2 d_{i}}{D^{2}} ; & 0 \leq d_{i} \leq D \\
0 ; & \text { otherwise }
\end{array} .\right.
$$

Then, from (59) and (61), we obtain the pdf of the $i$ th user's power at the receiver as

$$
f_{i}\left(G_{i}\right)=\left\{\begin{array}{cc}
\frac{2}{D^{2} A \alpha}\left(\frac{A}{G_{i}}\right)^{\frac{\alpha+2}{\alpha}} ; & G_{i} \geq A R^{-\alpha} \\
0 ; & \text { otherwise }
\end{array},\right.
$$

and the cumulative probability distribution function (CDF) as

$$
F_{i}\left(G_{i}\right)=\left\{\begin{array}{cc}
1-\left(\frac{A}{G_{i}}\right)^{\frac{2}{\alpha}} D^{-2} ; & G_{i} \geq A R^{-\alpha} \\
0 ; & \text { otherwise }
\end{array} .\right.
$$

Thus, the cost function is

$$
\begin{aligned}
c_{i} & =1-F_{i}\left(G_{i}\right)=\left(\frac{A}{G_{i}}\right)^{\frac{2}{\alpha}} D^{-2}, G_{i} \geq A R^{-\alpha} . \\
& =\left(\frac{d_{i}}{D}\right)^{2}, 0 \leq d_{i} \leq D
\end{aligned}
$$

In our simulation, $D=1$.

2) Numerical and Simulation Results: Figs. 9, 10, 11, and 12 present the numerical and simulation results for the MPR model. Figs. 9 and 10 show that the theoretical results match very well with the simulation results.

Figs. 11 and 12 compare the spectral efficiencies and energy consumption of different schemes. COOP-MUD-ALOHA based on the throughput optimization has almost the same performance as COOP-MUD-ALOHA based on the revenue optimization, which is much better than CONV-ALOHA. From the presented simulation results, we can see that our proposed COOP-MUD-ALOHA achieves much higher multiuser diversity gains (Figs. 11 and 12) compared with CONVALOHA, without sacrificing throughput or revenue (Figs. 9 and 10). However, different from the collision model, the throughput optimization and revenue optimization no longer yield identical throughput and revenue although the spectral efficiency and the energy consumption are almost the same. Therefore, there is a trade-off between COOP-MUD-ALOHA based on throughput optimization and COOP-MUD-ALOHA based on revenue optimization.

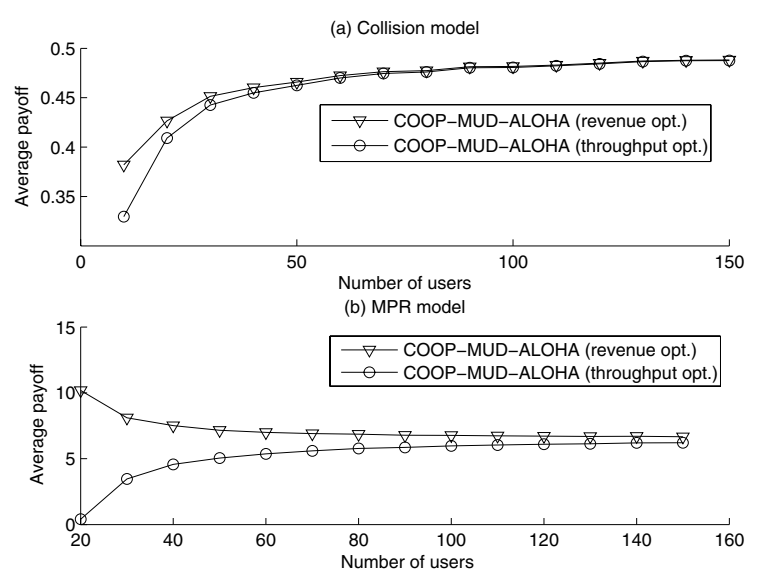

Fig. 8. Average expected payoff for collision and MPR models

\section{CONCLUSION}

In this paper, we study the problem of designing fair cooperative MAC schemes that achieve multiuser diversity gains. Based on a game-theoretic framework, we propose effective solutions for both the collision and MPR models. Throughput and revenue are investigated as performance metrics for the network. We show that if an appropriate pricing 


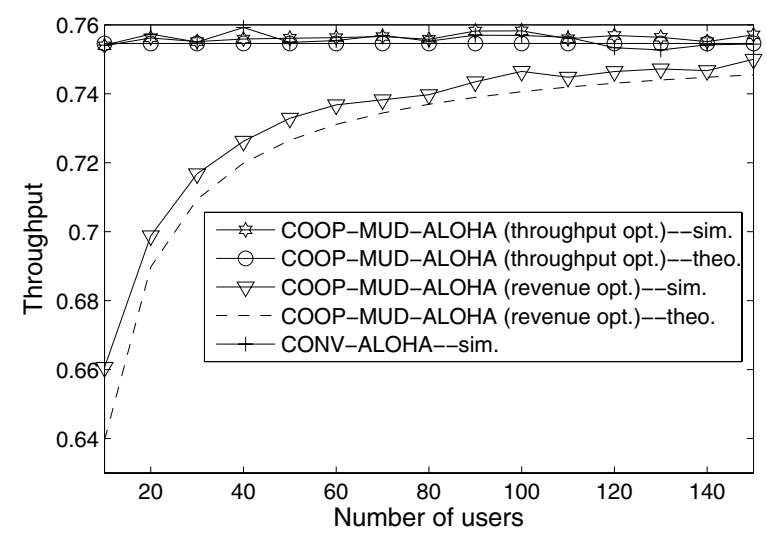

Fig. 9. Throughput comparison for the MPR model

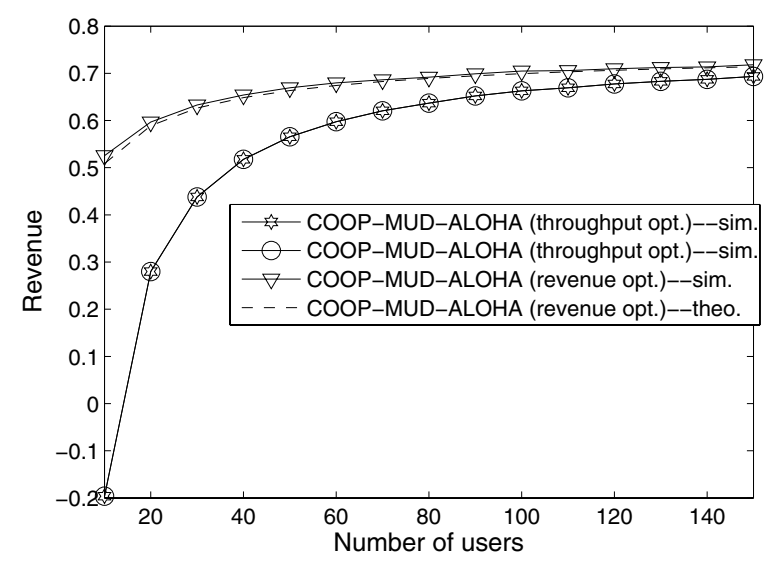

Fig. 10. Revenue comparison for the MPR model

is imposed, multiuser diversity gains can be achieved without sacrificing throughput or revenue for the network. Our results prove the viability of a distributed MAC implementation in wireless networks and determine the required policy to enforce cooperation and fairness in such networks in order to optimize the overall network performance.

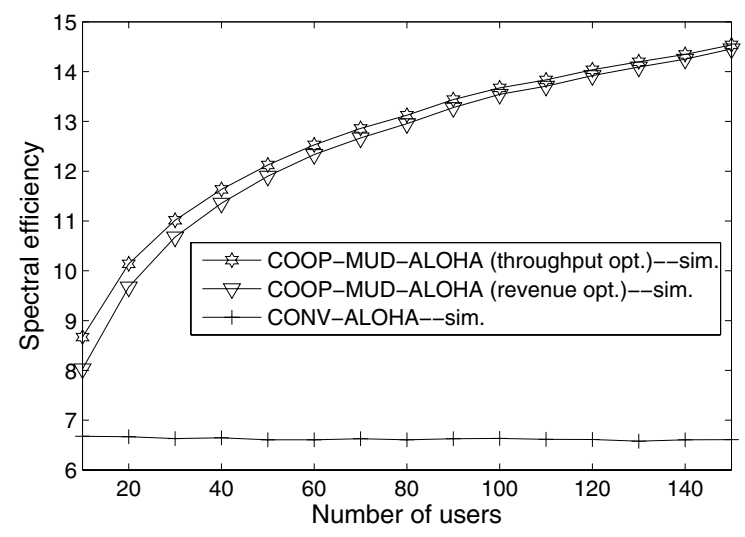

Fig. 11. Spectral efficiency comparison for the MPR model

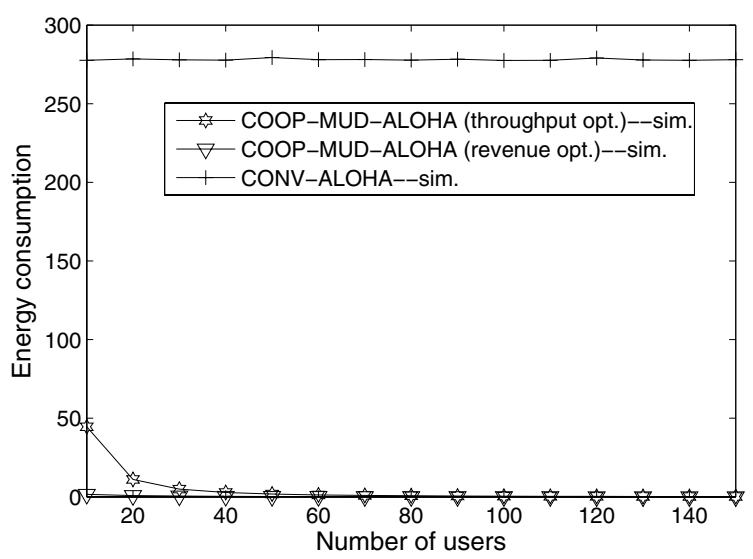

Fig. 12. Energy consumption comparison for the MPR model

\section{ACKNOWLEDGEMENT}

The authors would like to thank the anonymous reviewers and the editor for their careful review and constructive comments on this paper.

\section{REFERENCES}

[1] R. Knopp and P. A. Humblet, "Information capacity and power control in single-cell multiuser communications," in Proc. IEEE Int. Conf. Communications, Seattle, WA, Jun. 1995, pp. 331-335.

[2] D. N. C. Tse, "Optimal power allocation over parallel gaussian channels," in Proc. IEEE Int. Symp. Information Theory, Ulm, Germany, Jun. 1997, pp. 22-27.

[3] P. Viswanath, D. N. C. Tse, and R. Laroia, "Opportunistic beamforming using dumb antennas," IEEE Transactions on Information Theory, vol. 48, no. 6, pp. 1277-1294, Jun. 2002.

[4] X. Qin and R. Berry, "Exploiting multiuser diversity for medium access in wireless networks," in Proc. of IEEE INFOCOM Conf., vol. 2, San Francisco, CA, Apr. 2003, pp. 1084-1094.

[5] N. Feng, S. Mau, and N. B. Narayan, "pricing and power control for joint network-centric and user-centric radio resource management," IEEE Transactions on Communications, vol. 52, no. 9, pp. 1547-1557, Nov. 2004.

[6] E. Altman, D. Barman, R. E. Azouzi, and T. Jimenez, "A game theoretic approach for delay minimization in slotted aloha," in Proc. IEEE Int. Conf. Communications, vol. 7, Paris, Jun 2004, pp. 3999-4003.

[7] Y. Jin and G. Kesidis, "A pricing strategy for an aloha network of heterogeneous users with inelastic bandwidth requirements," in Proc. 39 th Annual Conference on Information Sciences and Systems, Princeton University, Mar. 2002.

[8] J. Sun and E. Modiano, "Opportunistic power allocation for fading channels with non-cooperative users and random access," in Proc. IEEE International Conference on Broadband Networks, vol. 1, Otc. 2005, pp. 366-374.

[9] R. D. Yates, "A framework for uplink power control in cellular radio systems," IEEE Journal on Selected Areas in Communications, vol. 13, pp. 1341-1347, Sept. 1995.

[10] E. Baccarelli, M. Biagi, and C. Pelizzoni, "A distributed powerallocation and signal-shaping game for the competitively optimal throughput-maximization of multiple-antenna "ad hoc" networks," IEEE Transactions on Vehicular Technology, vol. 55, pp. 1862-1876, Nov. 2004.

[11] A. B. MacKenzie and S. B. Wicker, "Selfish users in aloha: A gametheoretic approach," in Proc. IEEE Vehicular Technology Conference, vol. 3, Atlantic City, NJ, Oct. 2001, pp. 1354-1357.

[12] D. Wang, C. Comaniciu, and U. Tureli, "Cooperation and fairness for slotted aloha," Special Issue on Cooperation in Wireless Networks, Springer-Wireless Personal Communication, 2006.

[13] A. B. MacKenzie and S. B. Wicker, "Stability of multipacket slotted aloha with selfish users and perfect information," in Proc. IEEE INFOCOM, vol. 3, San Francisco, CA, Apr. 2003, pp. 1583-1590.

[14] D. Bertsekas and R. Gallager, Data Networks, 2nd ed. Prentice-Hall, Upper Saddle River, NJ, 1992. 
[15] D. Wang, C. Comaniciu, H. Minn, and N. Al-Dhahir, "On modeling utility for cooperative slotted aloha games," in IEEE ICASSP, Las Vegas, April 2008.

[16] S. Ghez, S. Verdu, and S. C. Schwartz, "Stability properties of slotted aloha with multipacket reception capability," IEEE Transactions on Automatic Control, vol. 33, no. 7, pp. 640-649, Feb. 1988.

[17] — "Optimal decentralized control in the random access multipacket channel," IEEE Transactions on Automatic Control, vol. 34, no. 11, pp. 640-649, Nov. 1989.

[18] G. Song and Y. Li, "Cross-layer optimization for OFDM wireless networks-part I: theoretical framework," IEEE Transactions on Wireless Communications, vol. 4, no. 2, pp. 614-624, Mar. 2005.

[19] H. Stark and J. W. Woods, Probability and random processes with applications to signal processing, 3rd ed. Prentice-Hall, Upper Saddle River, NJ, 2001

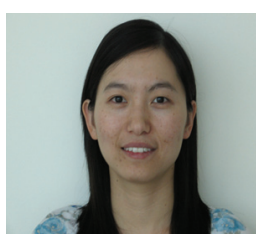

Dandan Wang (S'2005) received her B.S. and M.S degrees in electrical engineering from Beijing University of Posts \& Telecommunications, Beijing, China, in 2000 and 2003, respectively. She worked at China radio research lab, Ericsson from 2003 to 2004. From Aug, 2004 to Aug, 2005, she was with Department of Electrical and Computer Engineering, Stevens Institute of Technology, Hoboken, New Jersey. She is currently a Ph.D student at the Department of Electrical and Computer Engineering, University of Texas at Dallas. Her research interests include wireless communications and networking, signal processing for communications and sensor networks and cooperative/relay systems.

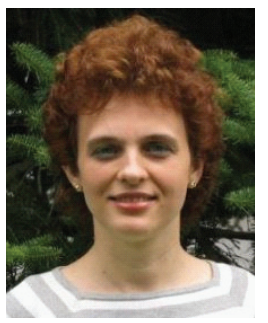

Cristina Comaniciu received the M.S. degree in electronics from the Polytechnic university of Bucharest in 1993, and the Ph.D. degree in electrical and computer engineering from Rutgers University in December 2001. From 1998 to 2001 she was with the Wireless Information Network Laboratory (WINLAB) at Rutgers University working towards her Ph.D. thesis. From 2002 to 2003 she was affiliated with the Electrical Engineering Department at Princeton University as a postdoctoral fellow. In August 2003, she joined Stevens Institute of Technology as an assistant professor and starting Fall 2008 she will be an associate professor at Stevens. She is a recipient of the WINSEC 2004 award for outstanding contributions, and a co-author of the book Wireless Networks: Multiuser Detection in Cross-Layer Design, Springer, NY, 2005. She recently won the 2007 IEEE Marconi Best Paper Prize Award in WiRELESS COMMUNiCATIONS for a paper co-authored with H. Vincent Poor. She currently serves as an Associate Editor for the sc IEEE Communications Letters Journal.

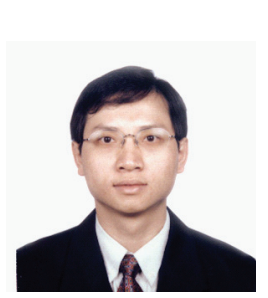

Hlaing Minn (S'99-M'01-SM'07) received his B.E. degree in Electronics from Yangon Institute of Technology, Yangon, Myanmar, in 1995, M.Eng. degree in Telecommunications from Asian Institute of Technology (AIT), Pathumthani, Thailand, in 1997 and $\mathrm{Ph} . \mathrm{D}$. degree in Electrical Engineering from the University of Victoria, Victoria, BC, Canada, in 2001. He was with the Telecommunications Program in AIT as a laboratory supervisor during 1998 . He was a research assistant from 1999 to 2001 and a post-doctoral research fellow during 2002 in the Department of Electrical and Computer Engineering at the University of Victoria. Since September 2002, he has been with the Erik Jonsson School of Engineering and Computer Science, the University of Texas at Dallas, USA, as an Assistant Professor. His research interests include wireless communications, statistical signal processing, error control, detection, estimation, synchronization, signal design, cross-layer design, cooperative/relay systems, and cognitive radios. He is an Editor for the IEEE TRANSACTIONS ON COMMUNICATIONS.

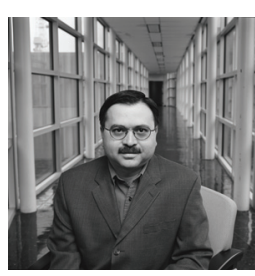

Naofal Al-Dhahir earned his $\mathrm{MS}$ and $\mathrm{PhD}$ degrees from Stanford University in 1990 and 1994, respectively, in Electrical Engineering. He was an Instructor at Stanford University in 1993.From 1994 to 1999 , he was a member of the technical staff at GE R\&D Center in NY where he worked on satellite communication systems design and antijam GPS receivers. From 1999 to 2003, he was a principal member of technical staff at AT\&T Shannon Laboratory in Florham Park, NJ where he worked on space-time coding and signal processing. In 2003, he joined UT-Dallas as an Associate Professor and became a ful Professor in 2007. He has served as a consultant to the Telecommunications Industry. His current research interests include space-time coding and signal processing, OFDM, wireless networks, and digital subscriber line technology. He has authored over 190 journal and conference papers and holds 22 US patents. He is a Fellow of the IEEE and a member of the IEEE SP4COM and SPTM technical committees. He served as Editor for IEEE TRANSACTION on Signal Processing and IEEE Communications LetTers and is currently an Editor for IEEE TRANSACTIONS ON COMMUNICATIONS. He served as co-chair of the Communication Theory Symposium at Globecom'04 and Tutorial Co-Chair for ICASSP'08. He is co-author of the book Doppler Applications for LEO Satellite Systems, Springer 2002. He is co-recipient of the IEEE VTC Fall 2005 best paper award, the 2005 IEEE signal processing society young author best paper award and the 2006 IEEE Donald G. Fink best paper award. 